Article

\title{
Comparing Lifetimes of Series and Parallel Systems with Heterogeneous Fréchet Components
}

\author{
Longxiang Fang ${ }^{*}+$ and Yanqin Wang $^{\dagger}$ \\ Department of Mathematics and Computer Science, Anhui Normal University, Wuhu 241000, China; \\ statistics201409@126.com \\ * Correspondence: lxfang@fudan.edu.cn; Tel.: +86-553-591-0757 \\ + These authors contributed equally to this work. \\ Academic Editor: Hari M. Srivastava \\ Received: 5 December 2016; Accepted: 6 January 2017; Published: 13 January 2017
}

\begin{abstract}
In this paper, we discuss stochastic comparisons of lifetimes of series and parallel systems with heterogeneous Fréchet components in terms of the usual stochastic order, reversed hazard rate order and likelihood ratio order. The partial results established here extend some well-known results in the literature of Gupta et al. Specifically, first, we generalize the result of Theorem 2 from the usual stochastic order to the reversed hazard rate order. Second, we generalize the result of Theorem 3 from the reversed hazard rate order to the likelihood ratio order. Last, we generalize the result of Theorem 4 from the hazard rate order to the likelihood ratio order when shape parameter $0<\alpha \leq 1$.
\end{abstract}

Keywords: Fréchet distribution; usual stochastic order; reversed hazard rate order; likelihood ratio order; series system; parallel system

\section{Introduction}

A random variable $X$ is said to have a Fréchet distribution if its cumulative distribution function (cdf) is

$$
F(x)=e^{-\left(\frac{x-\mu}{\theta}\right)^{-\alpha}}, x>\mu, \alpha>0, \theta>0,
$$

where $\mu, \theta$ and $\alpha$ are location, scale and shape parameters, respectively. We say that $X \sim$ Fré $(\mu, \theta, \alpha)$. The Fréchet distribution was introduced by Fréchet [1] as one of the extreme value distribution, and has been used in modeling and analysing several extreme events including accelerated life testing, earthquakes, wind speeds, and so on. A lot of studied results and various applications of the Fréchet distribution are presented in Broussard and Booth [2], Harlow [3], Kotz and Nadarajah [4], Xapson et al. [5], Gupta et al. [6], and so on.

Let $X_{1: n} \leq \ldots \leq X_{n: n}$ denote the order statistics corresponding to the random variables $X_{1}, \ldots, X_{n}$. These order statistics play an important role in reliability theory, operations research, auction theory, and many other areas; interested readers may refer to the volumes by Balakrishnan and Rao $[7,8]$ for relevant details. In particular, in reliability theory, the lifetime of a $k$-out-of- $n$ system is then evidently the $(n-k+1)$ th order statistic of a set of $n$ random variables representing the component lifetimes. So, a parallel (series) system is a $1(n)$-out-of- $n$ system and $X_{n: n}\left(X_{1: n}\right)$ denote its lifetime. Stochastic comparisons of parallel and series systems with heterogeneous components have been studied by many authors. For example, see Khaledi and Kochar [9,10], Genest et al. [11], Balakrishnan and Zhao [12], Balakrishnan et al. [13], and Gupta et al. [6].

Gupta et al. [6] have considered the stochastic comparisons between the lifetimes of parallel/series systems arising from independently distributed Fréchet components with respect to the usual stochastic 
order, the reversed hazard rate order and the hazard rate order based on location and scale parameters of the Fréchet distributed components.

In this paper, we first study the usual stochastic order comparison for the lifetimes of the parallel and series systems with independently Fréchet distributed components based on vector majorization of shape parameters but fixed location and scale parameters. Next, we generalize the corresponding results of Theorem 2, Theorem 3 and Theorem 4 in Gupta et al. [6]. Specifically, let $X_{1}, \ldots, X_{n}$ be independent random variables with $X_{i} \sim$ Fré $\left(\mu, \theta_{i}, \alpha_{i}\right), i=1, \ldots, n$, and $Y_{1}, \ldots, Y_{n}$ be independent random variables with $Y_{i} \sim \operatorname{Fré}\left(\mu, \theta_{i}^{*}, \alpha_{i}^{*}\right), i=1, \ldots, n$. Then:

(i) If $\theta_{1}=\ldots=\theta_{n}=\theta_{1}^{*}=\ldots=\theta_{n}^{*}$,

$$
\left(\alpha_{1}, \ldots, \alpha_{n}\right) \succeq_{m}\left(\alpha_{1}^{*}, \ldots, \alpha_{n}^{*}\right) \Longrightarrow X_{n: n} \geq_{s t} Y_{n: n} \text { and } X_{1: n} \leq_{s t} Y_{1: n}
$$

(ii) If $\alpha_{1}=\ldots=\alpha_{n}=\alpha_{1}^{*}=\ldots=\alpha_{n}^{*}$,

$$
\left(\frac{1}{\theta_{1}}, \ldots, \frac{1}{\theta_{n}}\right) \succeq^{w}\left(\frac{1}{\theta_{1}^{*}}, \ldots, \frac{1}{\theta_{n}^{*}}\right) \Longrightarrow X_{n: n} \geq_{r h} Y_{n: n} ;
$$

(iii) If $\alpha_{1}=\ldots=\alpha_{n}=\alpha_{1}^{*}=\ldots=\alpha_{n}^{*}$

$$
\sum_{i=1}^{n} \theta_{i}^{\alpha} \geq \sum_{i=1}^{n} \theta_{i}^{* \alpha} \Longrightarrow X_{n: n} \geq_{l r} Y_{n: n}
$$

(iv) If $\alpha_{1}=\ldots=\alpha_{n}=\alpha_{1}^{*}=\ldots=\alpha_{n}^{*}, \theta_{1}^{*}=\ldots=\theta_{n}^{*}=\theta$,

$$
\frac{1}{\theta}=\left(\prod_{i=1}^{n} \frac{1}{\theta_{i}}\right)^{\frac{1}{n}} \Longrightarrow X_{n: n} \geq_{l r} Y_{n: n} ;
$$

(v) If $\alpha_{1}=\ldots=\alpha_{n}=\alpha_{1}^{*}=\ldots=\alpha_{n}^{*}, \theta_{1}^{*}=\ldots=\theta_{n}^{*}=\theta^{*}$,

$$
\theta^{* \alpha}=\frac{1}{n} \sum_{i=1}^{n} \theta_{i}{ }^{\alpha} \text { or } 0<\alpha \leq 1, \theta^{*}=\frac{1}{n} \sum_{i=1}^{n} \theta_{i} \Longrightarrow X_{1: n} \leq_{l r} Y_{1: n}
$$

\section{Preliminaries}

Suppose the random variables $X$ and $Y$ have distribution functions $F(x)$ and $G(x)$, density functions $f(x)$ and $g(x)$, the survival functions $\bar{F}(x)=1-F(x)$ and $\bar{G}(x)=1-G(x)$, the hazard rate functions $r_{X}(x)=f(x) / \bar{F}(x)$ and $r_{Y}(x)=g(x) / \bar{G}(x)$, and the reversed hazard functions $\tilde{r}_{X}(x)=f(x) / F(x)$ and $\tilde{r}_{Y}(x)=g(x) / G(x)$, respectively. Several notions of stochastic orders, majorization and weak majorization have been discussed in Shaked and Shanthikumar [14] and Marshall et al. [15], and below we provide a basic description that are most relevant to the discussion here.

Definition 1. Let $X$ and $Y$ be two nonnegative random variables having support $(0,+\infty)$. Then:

(i) $X$ is said to be smaller than $Y$ in the reversed hazard rate order if $\tilde{r}_{X}(x) \leq \tilde{r}_{Y}(x)$, or equivalently, if $\frac{G(x)}{F(x)}$ is nondecreasing in $X$, and denoted by $Y \geq_{r h} X$;

(ii) $X$ is said to be smaller than $Y$ in the hazard rate order if $r_{X}(x) \geq r_{Y}(x)$, or equivalently, if $\frac{\bar{G}(x)}{\bar{F}(x)}$ is nondecreasing in $x$, and denoted by $Y \geq_{h r} X$;

(iii) $X$ is said to be smaller than $Y$ in the likelihood ratio order if $\frac{g(x)}{f(x)}$ is nondecreasing in $x$, and denoted by $Y \geq{ }_{l r} X$; 
(iv) $X$ is said to be smaller than $Y$ in the usual stochastic order if $\bar{G}(x) \geq \bar{F}(x)$, and denoted by $Y \geq_{s t} X$.

Definition 2. Let $\lambda=\left(\lambda_{1}, \ldots, \lambda_{n}\right)$ and $\lambda^{*}=\left(\lambda_{1}^{*}, \ldots, \lambda_{n}^{*}\right)$ be two real vectors, and $\lambda_{(1)} \leq \ldots \leq \lambda_{(n)}$ and $\lambda_{(1)}^{*} \leq \ldots \leq \lambda_{(n)}^{*}$ denote their ordered components. Then:

(1) $\quad \lambda^{*}$ is said to be majorized by $\lambda$, denoted by $\lambda \succeq_{m} \lambda^{*}$, if

$$
\sum_{i=1}^{j} \lambda_{(i)} \leq \sum_{i=1}^{j} \lambda_{(i)}^{*}
$$

for $j=1,2, \ldots, n-1$, and $\sum_{i=1}^{n} \lambda_{i}=\sum_{i=1}^{n} \lambda_{i}^{*} ;$

(2) $\lambda^{*}$ is said to be weak upper majorized by $\lambda$, denoted by $\lambda \succeq^{w} \lambda^{*}$, if

$$
\sum_{i=1}^{j} \lambda_{(i)} \leq \sum_{i=1}^{j} \lambda_{(i)}^{*}
$$

for $j=1,2, \ldots, n-1$, and $\sum_{i=1}^{n} \lambda_{i} \leq \sum_{i=1}^{n} \lambda_{i}^{*}$.

Before we present our main results, we need the following well-known concept and four lemmas.

Definition 3. Let $\lambda=\left(\lambda_{1}, \ldots, \lambda_{n}\right)$ and $\mu=\left(\mu_{1}, \ldots, \mu_{n}\right)$ be two real vectors. A real-valued function $\phi(\lambda): R^{n} \rightarrow R$ is said to be a Schur-concave (Schur-convex) function if for all $\lambda \succeq_{m} \mu$, we have $\phi(\lambda) \leq(\geq) \phi(\boldsymbol{\mu})$.

Lemma 1 (Marshall et al. [15]). A permutation-symmetric differentiable function $\phi(\boldsymbol{X})$ is Schur-concave (Schur-convex) if and only if

$$
\left(X_{i}-X_{j}\right)\left(\frac{\partial \phi(X)}{\partial X_{i}}-\frac{\partial \phi(X)}{\partial X_{j}}\right) \leq 0(\geq 0)
$$

for all $i \neq j$.

Lemma 2 (Marshall et al. [15]). Consider the real-valued function $\psi$, defined on a set $\mathbb{A} \subseteq R^{n}$. Then, $\boldsymbol{\mu} \succeq^{w} v$ implies $\psi(\boldsymbol{\mu}) \geq \psi(\boldsymbol{v})$ if and only if $\psi$ is nonincreasing and Schur-convex on $\mathbb{A}$.

Lemma 3 (Marshall et al. [15]). If $I \subset R$ is an interval and $h(x): I \rightarrow R$ is convex, then

$$
g(\boldsymbol{X})=\sum_{i=1}^{n} h\left(x_{i}\right)
$$

is Schur-convex on $I^{n}$, where $\boldsymbol{X}=\left(x_{1}, \ldots, x_{n}\right)$. Consequently, $\boldsymbol{Y}=\left(y_{1}, \ldots, y_{n}\right) \succeq_{m} \boldsymbol{X}=\left(x_{1}, \ldots, x_{n}\right)$ on $I^{n}$ implies $g(\boldsymbol{Y}) \geq g(\boldsymbol{X})$.

Lemma 4. Let the function $h:(0,+\infty) \times(0,+\infty) \rightarrow(-\infty,+\infty)$ be defined as

$$
h(y, t)=\frac{y^{-t} \ln y}{1-e^{y^{-t}}} .
$$

Then, for each $y>0, h(y, t)$ is nonincreasing with respect to $t$. 
Proof. For each fixed $y>0$, we have

$$
\frac{\partial h(y, t)}{\partial t}=\frac{(\ln y)^{2} y^{-t}\left(e^{y^{-t}}-1-y^{-t} e^{y^{-t}}\right)}{\left(1-e^{y^{-t}}\right)^{2}} .
$$

It is easy to verify that the function $e^{y^{-t}}-1-y^{-t} e^{y^{-t}}<0$ for any $t>0$. So we get $\frac{\partial h(y, t)}{\partial t}<0$ for any $t>0$, which implies that $h(y, t)$ is nonincreasing with respect to $t$

\section{Results}

First, we present the usual stochastic order comparison for the lifetimes of the parallel and series systems with independently Fréchet distributed components based on vector majorization of shape parameters but fixed location and scale parameters.

Theorem 1. Let $X_{1}, \ldots, X_{n}$ be independent random variables with $X_{i} \sim \operatorname{Fré}\left(\mu, \theta, \alpha_{i}\right), i=1, \ldots, n$, and $Y_{1}, \ldots, Y_{n}$ be independent random variables with $Y_{i} \sim \operatorname{Fré}\left(\mu, \theta, \alpha_{i}^{*}\right), i=1, \ldots, n$. If $\left(\alpha_{1}, \ldots, \alpha_{n}\right) \succeq_{m}$ $\left(\alpha_{1}^{*}, \ldots, \alpha_{n}^{*}\right)$, then $X_{n: n} \geq_{s t} Y_{n: n}$ and $X_{1: n} \leq_{s t} Y_{1: n}$.

Proof. (1) To prove that $X_{n: n} \geq_{s t} Y_{n: n}$, it is sufficient to prove, for $x>\mu$, that the cumulative distribution function

$$
F_{X_{n: n}}(x)=\prod_{i=1}^{n} e^{-\left(\frac{x-\mu}{\theta}\right)^{-\alpha_{i}}}=e^{-\sum_{i=1}^{n}\left(\frac{x-\mu}{\theta}\right)^{-\alpha_{i}}}
$$

is Schur-concave with respect to $\left(\alpha_{1}, \ldots, \alpha_{n}\right)$.

We have the derivative of $F_{X_{n: n}}(x)$ with respect to $\alpha_{i}, i=1, \ldots, n$, as, for $x>\mu$,

$$
\frac{\partial F_{X_{n: n}}(x)}{\partial \alpha_{i}}=F_{X_{n: n}}(x)\left(\frac{x-\mu}{\theta}\right)^{-\alpha_{i}} \ln \frac{x-\mu}{\theta} .
$$

Thus, we have

$$
\begin{aligned}
& \left(\alpha_{i}-\alpha_{j}\right)\left(\frac{\partial F_{X_{n: n}}(x)}{\partial \alpha_{i}}-\frac{\partial F_{X_{n: n}}(x)}{\partial \alpha_{j}}\right) \\
& =\left(\alpha_{i}-\alpha_{j}\right) F_{X_{n: n}}(x)\left[\left(\frac{x-\mu}{\theta}\right)^{-\alpha_{i}}-\left(\frac{x-\mu}{\theta}\right)^{-\alpha_{j}}\right] \ln \frac{x-\mu}{\theta} .
\end{aligned}
$$

If $\frac{x-\mu}{\theta} \geq 1$, then $\ln \frac{x-\mu}{\theta} \geq 0$ and $\left(\frac{x-\mu}{\theta}\right)^{-\alpha}$ is nonincreasing in $\alpha$. So, we obtain $\left(\alpha_{i}-\alpha_{j}\right)\left(\frac{\partial F_{X_{n: n}}(x)}{\partial \alpha_{i}}-\frac{\partial F_{X_{n: n}}(x)}{\partial \alpha_{j}}\right) \leq 0$;

If $0<\frac{x-\mu}{\theta}<1$, then $\ln \frac{x-\mu}{\theta}<0$ and $\left(\frac{x-\mu}{\theta}\right)^{-\alpha}$ is nonnonincreasing in $\alpha$. So, we obtain $\left(\alpha_{i}-\alpha_{j}\right)\left(\frac{\partial F_{X_{n: n}}(x)}{\partial \alpha_{i}}-\frac{\partial F_{X_{n: n}}(x)}{\partial \alpha_{j}}\right) \leq 0$.

Thus, upon using Lemma 1, we have $F_{X_{n: n}}(x)$ to be a Schur-concave function with respect to $\left(\alpha_{1}, \ldots, \alpha_{n}\right)$, which completes the proof.

(2) To prove that $X_{1: n} \leq_{s t} Y_{1: n}$, it is sufficient to prove, for $x>\mu$, that the survival function

$$
\bar{F}_{X_{1: n}}(x)=\prod_{i=1}^{n}\left(1-e^{-\left(\frac{x-\mu}{\theta}\right)^{-\alpha_{i}}}\right)
$$

is Schur-concave with respect to $\left(\alpha_{1}, \ldots, \alpha_{n}\right)$. 
We have the derivative of $\bar{F}_{X_{1: n}}(x)$ with respect to $\alpha_{i}, i=1, \ldots, n$, as, for $x>\mu$,

$$
\frac{\partial \bar{F}_{X_{1: n}}(x)}{\partial \alpha_{i}}=\bar{F}_{X_{1: n}}(x) \frac{e^{-\left(\frac{x-\mu}{\theta}\right)^{-\alpha_{i}}}\left(\frac{x-\mu}{\theta}\right)^{-\alpha_{i}}\left(-\ln \frac{x-\mu}{\theta}\right)}{1-e^{-\left(\frac{x-\mu}{\theta}\right)^{-\alpha_{i}}}} .
$$

Thus, we have

$$
\begin{aligned}
& \left(\alpha_{i}-\alpha_{j}\right)\left(\frac{\partial \bar{F}_{X_{1: n}}(x)}{\partial \alpha_{i}}-\frac{\partial \bar{F}_{X_{1: n}}(x)}{\partial \alpha_{j}}\right) \\
& =\left(\alpha_{i}-\alpha_{j}\right) \bar{F}_{X_{1: n}}(x)\left[\frac{\left(\frac{x-\mu}{\theta}\right)^{-\alpha_{i}} \ln \frac{x-\mu}{\theta}}{1-e^{\left(\frac{x-\mu}{\theta}\right)^{-\alpha_{i}}}}-\frac{\left(\frac{x-u}{\theta}\right)^{-\alpha_{j}} \ln \frac{x-\mu}{\theta}}{1-e^{\left(\frac{x-\mu}{\theta}\right)^{-\alpha_{j}}}}\right] \\
& \leq 0 .
\end{aligned}
$$

The last inequality holds according to Lemma 4 , that is, $\bar{F}_{X_{1: n}}(x)$ is a Schur-concave function with respect to $\left(\alpha_{1}, \ldots, \alpha_{n}\right)$, which completes the proof.

Now, we discuss stochastic comparison of the lifetimes of parallel systems having independently Fréchet distributed components with respect to the reversed hazard rate order based on vector weak upper majorization of scale parameters but fixed location and shape parameters. The following result generalizes the result of Theorem 2 in Gupta et al. [6].

Theorem 2. Let $X_{1}, \ldots, X_{n}$ be independent random variables with $X_{i} \sim$ Fré $\left(\mu, \theta_{i}, \alpha\right), i=1, \ldots, n$, and $Y_{1}, \ldots, Y_{n}$ be independent random variables with $Y_{i} \sim \operatorname{Fré}\left(\mu, \theta_{i}^{*}, \alpha\right), i=1, \ldots, n$. If $\left(\frac{1}{\theta_{1}}, \ldots, \frac{1}{\theta_{n}}\right) \succeq^{w}$ $\left(\frac{1}{\theta_{1}^{*}}, \ldots, \frac{1}{\theta_{n}^{*}}\right)$, then $X_{n: n} \geq_{r h} Y_{n: n}$.

Proof. By equation (1), the probability density function of $X_{n: n}$, for $x>\mu$, is

$$
f_{X_{n: n}}(x)=F_{X_{n: n}}(x) \sum_{i=1}^{n} \frac{\alpha}{x-\mu}\left(\frac{x-\mu}{\theta_{i}}\right)^{-\alpha} .
$$

So, the reversed hazard function of $X_{n: n}$ is

$$
\tilde{r}_{X_{n: n}}(x)=\sum_{i=1}^{n} \frac{\alpha}{x-\mu}\left(\frac{x-\mu}{\theta_{i}}\right)^{-\alpha} .
$$

Let $t_{i}=\frac{1}{\theta_{i}}, t_{i}^{*}=\frac{1}{\theta_{i}^{*}}$, then, we have

$$
\tilde{r}_{X_{n: n}}(x)=\sum_{i=1}^{n} h\left(t_{i}\right)
$$

where $h(t)=\frac{\alpha}{x-\mu}(t(x-\mu))^{-\alpha}$.

It is obvious that the function $h(t)$ is nonincreasing and convex in $t$. So, for $x>\mu$, we have $\tilde{r}_{X_{n: n}}(x)$ is nonincreasing and Schur-convex in $\left(t_{1}, \ldots, t_{n}\right)$ by Lemma 3. That is, $\left(t_{1}, \ldots, t_{n}\right) \succeq^{w}\left(t_{1}^{*}, \ldots, t_{n}^{*}\right)$ implies $\tilde{r}_{X_{n: n}} \geq \tilde{r}_{Y_{n: n}}$ by Lemma 2 . This completes the proof of the theorem.

Next, we present stochastic comparison of the lifetimes of parallel systems having independently Fréchet distributed components with respect to the likelihood ratio order based on different scale parameters but fixed location and shape parameters. 
Theorem 3. Let $X_{1}, \ldots, X_{n}$ be independent random variables with $X_{i} \sim$ Fré $\left(\mu, \theta_{i}, \alpha\right), i=1, \ldots, n$, and $Y_{1}, \ldots, Y_{n}$ be independent random variables with $Y_{i} \sim \operatorname{Fré}\left(\mu, \theta_{i}^{*}, \alpha\right), i=1, \ldots, n$. If $\sum_{i=1}^{n} \theta_{i}^{\alpha} \geq \sum_{i=1}^{n} \theta_{i}^{* \alpha}$, then $X_{n: n} \geq_{l r} Y_{n: n}$.

Proof. By Equations (1) and (3), the probability density function $f_{X_{n: n}}(x)$, for $x>\mu$, can be written as,

$$
f_{X_{n: n}}(x)=\alpha(x-\mu)^{-\alpha-1} \sum_{i=1}^{n} \theta_{i}^{\alpha} e^{-\sum_{i=1}^{n} \theta_{i}^{\alpha}(x-\mu)^{-\alpha}}
$$

Similarly, the density function of $Y_{n: n}$, for $x>\mu$, is given by

$$
g Y_{n: n}(x)=\alpha(x-\mu)^{-\alpha-1} \sum_{i=1}^{n} \theta_{i}^{* \alpha} e^{-\sum_{i=1}^{n} \theta_{i}^{* \alpha}(x-\mu)^{-\alpha}} .
$$

Then, the ratio of the density functions of $X_{n: n}$ and $Y_{n: n}$, for $x>\mu$, can be shown as,

$$
\frac{f_{X_{n: n}}(x)}{g Y_{n: n}(x)}=\frac{\sum_{i=1}^{n} \theta_{i}^{\alpha}}{\sum_{i=1}^{n} \theta_{i}^{* \alpha}} e^{(x-\mu)^{-\alpha}\left(\sum_{i=1}^{n} \theta_{i}^{* \alpha}-\sum_{i=1}^{n} \theta_{i}^{\alpha}\right)} .
$$

Thus, if $\sum_{i=1}^{n} \theta_{i}^{\alpha} \geq \sum_{i=1}^{n} \theta_{i}^{* \alpha}$, we have $\frac{f_{X_{n: n}}(x)}{\delta_{X_{n: n}}(x)}$ is nonnonincreasing for $x>\mu$. Hence, the theorem.

The following result provides the likelihood ratio order comparison between the largest order statistics from independent heterogeneous Fréchet random variables and i.i.d. Fréchet random variables. The established result generalizes the result of Theorem 3 in Gupta et al. [6] from the reversed hazard rate order to the likelihood ratio order.

Theorem 4. Let $X_{1}, \ldots, X_{n}$ be independent random variables with $X_{i} \sim$ Fré $\left(\mu, \theta_{i}, \alpha\right), i=1, \ldots, n$, and $Y_{1}, \ldots, Y_{n}$ be independent random variables with $Y_{i} \sim$ Fré $(\mu, \theta, \alpha), i=1, \ldots, n$. If $\frac{1}{\theta}=\left(\prod_{i=1}^{n} \frac{1}{\theta_{i}}\right)^{\frac{1}{n}}$, then $X_{n: n} \geq_{l r} Y_{n: n}$.

Proof. It is obvious that the probability density functions of $X_{n: n}$ and $Y_{n: n}$, for all $x>\mu$, can be written as,

$$
f_{X_{n: n}}(x)=F_{X_{n: n}}(x) \sum_{i=1}^{n} \frac{\alpha}{x-\mu}\left(\frac{x-\mu}{\theta_{i}}\right)^{-\alpha}
$$

and

$$
g Y_{n: n}(x)=F_{Y_{n: n}}(x) \frac{n \alpha}{x-\mu}\left(\frac{x-\mu}{\theta}\right)^{-\alpha}
$$

respectively. Therefore, the ratio of the density functions of $X_{n: n}$ and $Y_{n: n}$, for $x>\mu$, is

$$
\frac{f_{X_{n: n}}(x)}{g_{Y_{n: n}}(x)}=\frac{F_{X_{n: n}}(x)}{F_{Y_{n: n}}(x)} \frac{\sum_{i=1}^{n}\left(\frac{1}{\theta_{i}}\right)^{-\alpha}}{n\left(\frac{1}{\theta}\right)^{-\alpha}}
$$

From the result of Theorem 3 in Gupta et al. [6], we have $\frac{1}{\theta}=\left(\prod_{i=1}^{n} \frac{1}{\theta_{i}}\right)^{\frac{1}{n}}$ implies $X_{n: n} \geq_{r h} Y_{n: n,}$ that is, $\frac{F_{X_{n: n}}(x)}{F_{Y_{n: n}}(x)}$ is nondecreasing for $x>\mu$. So, $\frac{f_{X_{n: n}}(x)}{g_{Y_{n: n}}(x)}$ is nondecreasing for $x>\mu$, the desired result is obtained.

Last, we discuss stochastic comparison of the lifetimes of two series systems, which having independently heterogeneous Fréchet distributed components and i.i.d. Fréchet distributed 
components respectively, with respect to the likelihood ratio order based on different scale parameters but fixed location and shape parameters. We first present another sufficient condition on stochastic comparison of the lifetimes of series systems having independently Fréchet distributed components with respect to the likelihood ratio order when shape parameter $0<\alpha \leq 1$.

Lemma 5. Let $X_{1}, \ldots, X_{n}$ be independent random variables with $X_{i} \sim$ Fré $\left(\mu, \theta_{i}, \alpha\right), i=1, \ldots, n$, and $Y_{1}, \ldots, Y_{n}$ be independent random variables with $Y_{i} \sim$ Fré $\left(\mu, \theta^{*}, \alpha\right), i=1, \ldots, n$. If $0<\alpha \leq 1$ and $\left(\theta_{1}, \ldots, \theta_{n}\right) \succeq_{m}\left(\theta_{1}^{*}, \ldots, \theta_{n}^{*}\right)$, then $X_{1: n} \leq_{l r} Y_{1: n}$.

Proof. By Equation (2), the hazard rate function of $X_{1: n}$, for all $x>\mu$, can be written as,

$$
r_{X_{1: n}}(x)=\frac{\alpha}{x-\mu} \sum_{i=1}^{n} \frac{\left(\frac{x-\mu}{\theta_{i}}\right)^{-\alpha}}{e^{\left(\frac{x-\mu}{\theta_{i}}\right)^{-\alpha}}-1} .
$$

According to the proof of Theorem 4 in [6], we see the function $\frac{u_{i}}{e^{u_{i}}-1}$ is nonincreasing and convex in $u_{i}$ for $u_{i} \in(0, \infty), i=1, \ldots, n$. So, the composite function $\sum_{i=1}^{n} \frac{\left(\frac{x-\mu}{\theta_{i}}\right)^{-\alpha}}{e^{\left(\frac{x-\mu}{\theta_{i}}\right)^{-\alpha}}-1}$ is convex in $\theta_{i}$ for $0<\alpha \leq 1$ and $\theta_{i} \in(0, \infty), i=1, \ldots, n$. Thus, for $x>\mu$, we have $r_{X_{1: n}}(x)$ is Schur-convex in $\left(\theta_{1}, \ldots, \theta_{n}\right)$ by Lemma 3 , that is, if $\left(\theta_{1}, \ldots, \theta_{n}\right) \succeq_{m}\left(\theta_{1}^{*}, \ldots, \theta_{n}^{*}\right)$ implies $r_{X_{1: n}} \geq r_{Y_{1: n}}$ by Lemma 2 , which completes the proof.

Theorem 5. Let $X_{1}, \ldots, X_{n}$ be independent random variables with $X_{i} \sim$ Fré $\left(\mu, \theta_{i}, \alpha\right), i=1, \ldots, n$, and $Y_{1}, \ldots, Y_{n}$ be independent random variables with $Y_{i} \sim \operatorname{Fré}\left(\mu, \theta^{*}, \alpha\right), i=1, \ldots, n$.

(1) If $\theta^{* \alpha}=\frac{1}{n} \sum_{i=1}^{n} \theta_{i}{ }^{\alpha}$, then $X_{1: n} \leq_{l r} Y_{1: n}$;

(2) If $0<\alpha \leq 1, \theta^{*}=\frac{1}{n} \sum_{i=1}^{n} \theta_{i}$, then $X_{1: n} \leq_{l r} Y_{1: n}$.

Proof. (1) By equation (2), we obtain the probability density functions of $X_{1: n}$ and $Y_{1: n}$, for all $x>\mu$, can be written as,

$$
f_{X_{1: n}}(x)=\bar{F}_{X_{1: n}}(x) \frac{\alpha}{x-\mu} \sum_{i=1}^{n} \frac{\left(\frac{x-\mu}{\theta_{i}}\right)^{-\alpha}}{e^{\left(\frac{x-\mu}{\theta_{i}}\right)^{-\alpha}}-1}
$$

and

$$
g Y_{1: n}(x)=\bar{F}_{Y_{1: n}}(x) \frac{n \alpha}{x-\mu} \frac{\left(\frac{x-\mu}{\theta^{*}}\right)^{-\alpha}}{e^{\left(\frac{x-\mu}{\theta^{*}}\right)^{-\alpha}}-1}
$$

respectively.

In order to prove that $X_{1: n} \leq_{l r} Y_{1: n}$, it is sufficient to prove that the ratio of density functions

$$
\frac{g Y_{1: n}(x)}{f_{X_{1: n}}(x)}=\frac{\bar{F}_{Y_{1: n}}(x)}{\bar{F}_{X_{1: n}}(x)} \frac{\frac{n\left(\frac{x-\mu}{\theta^{*}}\right)^{-\alpha}}{e^{\left(\frac{x-\mu}{\theta^{*}}\right)^{-\alpha}-1}}}{\sum_{i=1}^{n} \frac{\left(\frac{x-\mu}{\theta_{i}}\right)^{-\alpha}}{e^{\left(\frac{x-\mu}{\theta_{i}}\right)^{-\alpha}-1}}} \triangleq \frac{\bar{F}_{Y_{1: n}}(x)}{\bar{F}_{X_{1: n}}(x)} \eta(x)
$$

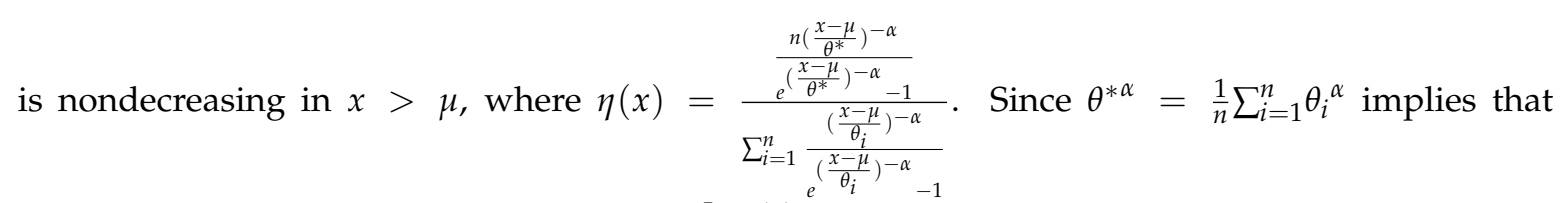

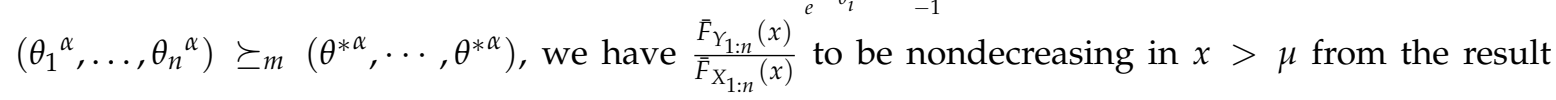


of Theorem 4 in Gupta et al. [6]. So it suffices to show that $\eta(x)$ is nondecreasing in $x>\mu$. Without loss of generality, taking $\mu=0$, then we have $\eta(x)=\frac{\frac{n \theta^{* \alpha}}{e^{\theta^{* \alpha} x^{-\alpha}}-1}}{\sum_{i=1}^{n} \frac{\theta_{i}^{\alpha}}{e^{\theta_{i}^{\alpha}} x^{-\alpha}-1}}, x>0$. According to the proof of Theorem 2 in Fang and Balakrishnan [16], we see that $\frac{\frac{n \lambda^{*}}{e^{*} y}-1}{\sum_{i=1}^{n} \frac{\lambda_{i}}{e^{\lambda_{i} y}-1}}$ is nonincreasing in $y>0$ with $\lambda^{*}=\frac{1}{n} \sum_{i=1}^{n} \lambda_{i}$. Thus, we have $\frac{\frac{n \theta^{* \alpha}}{e^{\theta^{* \alpha}} y-1}}{\sum_{i=1}^{n} \frac{\theta_{i}^{\alpha}}{e^{\theta_{i}}{ }^{\alpha} y}}$ is nonincreasing in $y>0$ with $\theta^{* \alpha}=\frac{1}{n} \sum_{i=1}^{n} \theta_{i}{ }^{\alpha}$. Also, $x^{-\alpha}$ is a nonincreasing function in $x>0$. So, the composite function $\eta(x)$ is an nondecreasing function in $x>0$, and the required result then follows.

(2) Upon using Lemma 5, this proof is similar to that of Part (1), and hence is not presented here for the sake of conciseness.

\section{Conclusions}

In a process the stochastic behavior of extreme values may be charactered by using extreme value theory. Fréchet distribution is one of the extreme value distribution, called extreme value type-II distribution, derived by Fréchet [1] in 1927. Gupta et al. [6] have considered the stochastic comparisons between the lifetimes of parallel/series systems arising from independently distributed Fréchet components with respect to the usual stochastic order, the reversed hazard rate order and the hazard rate order based on location and scale parameters of the Fréchet distributed components. In this paper, we generalize some results established in the Gupta et al. [6]. Specifically, first, the established result in Theorem 3 generalizes the result of Theorem 2 in Gupta et al. [6] from the usual stochastic order to the reversed hazard rate order. Second, the established result in Theorem 4 generalizes the result of Theorem 3 in Gupta et al. [6] from the reversed hazard rate order to the likelihood ratio order. Last, the established result in Theorem 5 generalizes the result of Theorem 4 in Gupta et al. [6] from the hazard rate order to the likelihood ratio order when shape parameter $0<\alpha \leq 1$. Since Fréchet distribution has become one of the popular lifetime models in reliability literature and that we discuss stochastic orderings for largest and smallest order statistics, the results established directly relate to some key distributional properties and features of parallel and series systems, two most common coherent systems, with Fréchet components. Furthermore, these results may also be useful in establishing some statistical properties of estimators of the scale and shape parameters of the Fréchet distribution. We are currently looking into this problem and hope to report the findings in a future paper.

Acknowledgments: This research was partially supported by National Natural Science Foundation of China (No. 11201003), the Provincial Natural Science Research Project of Anhui Colleges (No. KJ2016A263), the National Natural Science Foundation of Anhui Province (No. 1408085MA07), and the PhD research startup foundation of Anhui Normal University (No. 2014bsqdjj34).

Author Contributions: Longxiang Fang and Yanqin Wang conceived and designed this paper, and contributed equally to this work.

Conflicts of Interest: The authors declare no conflict of interest. The founding sponsors had no role in the design of the study; in the collection, analyses, or interpretation of data; in the writing of the manuscript, and in the decision to publish the results.

\section{References}

1. Fréchet, M. Sur la loi de probabilité de lècart maximum. Ann. Soc. Pol. Math. Crac. 1927, 6, 93-117.

2. Broussard, J.P.; Booth, G.G. The behavior of extreme values in Germany's stock index futures: An application to intradaily margin setting. Eur. J. Oper. Res. 1988, 104, 393-402.

3. Harlow, D.G. Applications of the Fréchet distribution function. Int. J. Mater. Prod. Technol. 2002, 5, 482-495.

4. Kotz, S.; Nadarajah, S. Extreme Value Distributions: Theory and Applications; Imperial College Press: London, UK, 2000. 
5. Xapson, M.A.; Summers, G.P.; Barke, E.A. Extreme value analysis of solar energetic motion peak fluxes. Sol. Phys. 1998, 183, 157-164.

6. Gupta, N.; Patra, L.K.; Kumar, S. Stochastic comparisons in systems with Frechet distributed components. Oper. Res. Lett. 2015, 43, 612-615.

7. Balakrishnan, N.; Rao, C.R. (Eds.) Handbook of Statistics 16-Order Statistics: Theory and Methods; Elsevier: Amsterdam, The Netherlands, 1998.

8. Balakrishnan, N.; Rao, C.R. (Eds.) Handbook of Statistics 17-Order Statistics: Applications; Elsevier: Amsterdam, The Netherlands, 1998.

9. Khaledi, B.; Kochar, S.C. Some new results on stochastic comparisons of parallel systems. J. Appl. Probab. 2000, 37, 1123-1128.

10. Khaledi, B.; Kochar, S.C. Weibull distribution: Some stochastic comparisons results. J. Stat. Plan. Inference 2006, 136, 3121-3129.

11. Genest, C.; Kochar, S.C.; Xu, M. On the range of heterogeneous samples. J. Multivar. Anal. 2009, 100, 1587-1592.

12. Balakrishnan, N.; Zhao, P. Ordering properties of order statistics from heterogeneous populations: A review with an emphasis on some recent developments. Probab. Eng. Inf. Sci. 2013, 27, 403-469.

13. Balakrishnan, N.; Haidari, A.; Masoumifard, K. Stochastic comparisons of series and parallel systems with generalized exponential components. IEEE Trans. Reliab. 2015, 64, 333-348.

14. Shaked, M.; Shanthikumar, J.G. Stochastic Orders; Springer: New York, NY, USA, 2007.

15. Marshall, A.W.; Olkin, I.; Arnold, B.C. Inequalities: Theory of Majorization and Its Applications; Springer: New York, NY, USA, 2011.

16. Fang, L.; Balakrishnan, N. Likelihood ratio order of parallel systems with heterogeneous Weibull components. Metrika 2016, 79, 693-703.

(C) 2017 by the authors; licensee MDPI, Basel, Switzerland. This article is an open access article distributed under the terms and conditions of the Creative Commons Attribution (CC-BY) license (http://creativecommons.org/licenses/by/4.0/). 